\section{Zinc-Finger Proteins in Brain Development and Mental Illness}

\section{Abstract}

Current genomic screening technologies race to screen genes involved in mental illness in large population or case by case patients including schizophrenia, bipolar disease, depression, autism spectrum disorders and intellectual disability. Among identified genes, zinc finger domain (ZNF/zfp) containing proteins come to attention. Copy number variant (CNV), single nucleotide polymorphism (SNP) as well as de novo mutations induced nonsense or missense mutations in proteins, have been found in a handful ZNF/zfp genes, much mutations are within or near zinc finger domains. Those genes are found to be expressed during early embryonic and postnatal development. Nevertheless, studies on roles of ZNF/zfp domain containing proteins and brain development are necessary to reveal pathogenesis of human mental illness. Here in this review, we summarize recent discoveries on mental illness related ZNF/zfp genes and their role in brain development.

Keywords: Mental illness; Brain development; ZNF/zfp proteins; Genome

\section{Hong Li ${ }^{1 *}$, Meifang Lu² and Xiaoyan Liu ${ }^{2}$}

\author{
1 Department Neuroscience, Yale \\ University School of Medicine, New \\ Haven, CT 06520, USA \\ 2 Department of Histology and \\ Embryology, School of Basic Medical \\ Sciences, Anhui, Medical University, \\ Hefei, Anhui, China
}

\section{*Corresponding author: Hong Li \\ झ hong.li@yale.edu}

Ph.D, Department of Neuroscience, Yale University School of Medicine, New Haven, CT 06520, USA.

Tel: $516-851-8564$

Citation: Hong Li, Meifang Lu, Liu X (2018) Zinc-Finger Proteins in Brain Development and Mental Illness. J Transl Neurosci Vol.3 No.2:4

\section{Introduction}

ZNF/zfp protein family is a group of massive and diverse proteins containing small functional zinc finger domain(s) that requires coordination of at least one zinc iron to stabilize its structure [1,2]. Zinc finger domain is capable of binding DNA, RNA and proteins or small molecules and ZNF/zfp proteins usually consist of multiple zinc finger domains in combination to other functional domains, forming one of the most diverse protein families in the organism kingdom and playing important roles during biological evolution. ZNF/zfp proteins are involved in the regulation of several cellular processes, eg, transcriptional regulation, protein translation and folding, DNA replication and repair, chromatin remodeling, cell proliferation and migration, signal transduction and metabolism $[3,4]$. Interestingly, recent genome-wide association (GWAS) studies revealed that many ZNF/zfp genes are associated with mental illnesses such as schizophrenia, bipolar diseases and intellectual disability [3-7]. Gene expression and function studies of some of the genes associated with neuropsychiatric diseases found their expression in developing neocortex during early or middle term fetal development and play critical roles in differentiation and maturation of neocortical neurons. In this review, we briefly summarize recent advances in ZNF/zfp genes and their roles on cortical development and mental illnesses.

\section{ZNFs Classification, Structure and Functions}

Zinc finger structure can be classified into classical and nonclassical types according to their differences in cysteine/histidine combinations which coordinate zinc irons. The most abundant classical $\mathrm{C} 2 \mathrm{H} 2$ zinc finger domain contains two $\beta$-sheets and one $\alpha$-helix, and the majority of this type of proteins are transcription factors that recognize specific DNA sequence (T/A)(G/A) (CAGAA(T/G/C) [4]. Non-classical ZNF/zfp proteins comprise of Ring Finger domain (RING, C3HC4), plant homeo domain (PHD, C4HC3), LIM domains and others. Proteins containing RING domain usually involve in ubiquitination pathway, for example, ubiquitin E3 ligase [8]. PHD domain proteins are chromatinassociated proteins with ability to read and bind the methylated or acetylated forms of histone 3 (H3) [9]. The detailed classification of different combinations of cysteine/histidine in a zinger finger domain can be found in recent review by Cassandri et al. [4]. One of important characteristic of ZNF/zfp domain containing proteins is that they often have multiple zinger finger domains in combination with other types of protein motifs. For example, LIM 
family proteins can be classified into three subclasses:

(1) LIM-homeodomain proteins, such as Lhx family proteins $[10,11]$;

(2) LIM-functional domain proteins, for example LIM kinase;

(3) LIM-only proteins, Such as LMO1-LMO4, FHL1 $[12,13]$.

Due to the diversity of the zinc finger protein structure, it has a wide range of functions in the cell process such as transcriptional repression, chromatin remodeling, protein degradation, cell cycle progression and cell migration.

\section{Mental IIIness with ZNFs}

Recently, accumulated ZNF/zfp genes have been found to associate with cognitive defects through genome wide associated studies. FOXP2, a language disorder associated gene, has a zinc finger domain and a leucine zipper motif in addition to a forkhead domain and its transcriptional activity is regulated by homo- and heterodimerization via a zinc finger (Figure 1) and a leucine zipper motif [5]. FOXP2 is expressed in fetal brain as early as embryonic day 12 (E12) and persists into adulthood with high expression in basal ganglion, layer $\mathrm{VI}$ of neocortex, thalamus and cerebellar. There is no FOXP2 expression found in the ventricular zone of telencephalon, but present in migrating neurons as well as differentiated neurons [14]. Myelin transcription factor 1 like (MYT1L) is a C2HC zinc finger transcription factor whose expression is restricted to neuronal tissues, and peaks during neurogenesis [15]. In humans, it has one N-terminal zinc finger, two tandem central zinc fingers, and three C-terminal zinc fingers (Figure 1). Clinical studies showed that microduplications disrupting MYT1L gene are associated with increasing risk for childhood-onset schizophrenia [14]. Complete deletion, intragenic deletion, duplication and de novo point mutation in MYT1L gene has been found to be responsible for intellectual disability [15]. Notably, MYT1L is reported to represses genes with prominent non-neuronal developmental roles, but not neuronal lineage specification genes during fibroblasts reprograming to neurons, thus functions as a pro-neuronal gene [16].
ZBTB20 belongs to a family of transcription factors with an $\mathrm{N}$-terminal $\mathrm{BTB} / \mathrm{POZ}$ domain involved in protein-protein interaction and five $\mathrm{C} 2 \mathrm{H} 2$ zinc fingers at the $\mathrm{C}$ terminus (Figure 1), mediating protein binding to DNA [17]. ZBTB20 is expressed in developing neocortex and adult hippocampus. Studies on patients with major depressive disorder (MDD) found that hypermethylation within coding region of ZBTB20 gene is associated with the occurrence of MDD [18].

Primrose syndrome is a rare congenital genetic defect syndrome, characterized by intellectual disability, disturbed behavior, hypotonia, unusual facial features and increased postnatal growth. Whole-exome sequencing of 8 subjects with Primrose syndrome found missense mutations or microdeletion in ZBTB20 loci [17]. The mutation site of the ZBTB20 gene is conservative in all cases of Primrose syndrome, and the mutant amino acid residues are located in the first, second zinc finger region and the junction area of the zinc finger. In vitro experiments showed that the mutation of these loci reduced the binding ability of ZBTB20 protein to the target DNA fragments, and its ability of transcriptional inhibition was significantly reduced [17]. This finding establishes a genetic link between these disorders and delineates the impact of ZBTB20 dysregulation on brain development, cognitive function, growth and metabolism.

Recently, several genome-wide association studies (GWAS) suggested that a classical $\mathrm{C} 2 \mathrm{H} 2$-type zinc finger domain containing protein ZNF804a is one of the most susceptible genes for schizophrenia and bipolar disease $[19,20]$. Human ZNF804A has one $\mathrm{C} 2 \mathrm{H} 2$ domain at $\mathrm{N}^{\prime}$-terminal, which is supposed to bind DNA in a sequence-specific manner (Figure 1). A number of genetic variations, such as single nucleotide polymorphism (SNP) rs1344706 within the ZNF804A gene, are of risky variants for schizophrenia in European populations [20,21]. But a recent study showed inconformity with the results in Han Chinese [22]. The inconsistency between studies in Europeans and Chinese populations may be mainly caused by different genetic backgrounds, the allelic frequencies of many SNPs could vary across different populations. In addition to schizophrenia and

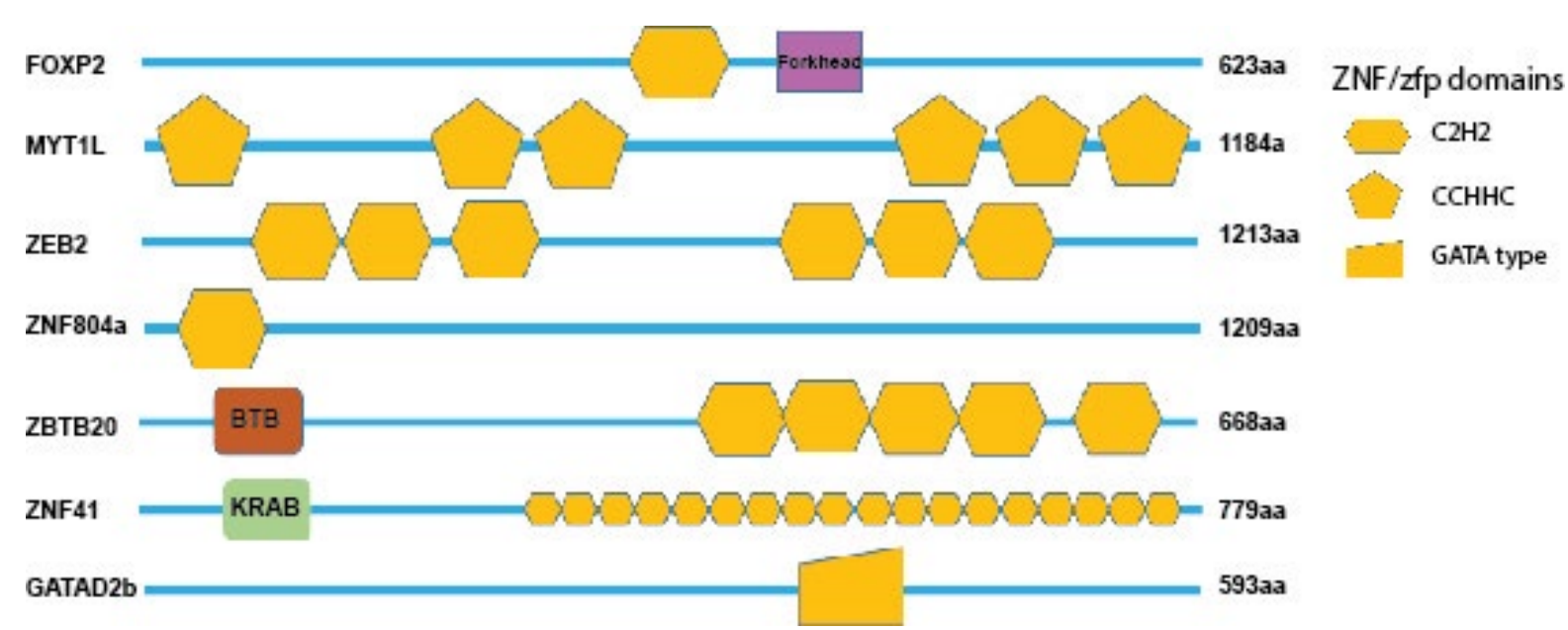

Figure 1 Diagrams of protein domains of ZNF/zfp genes associated with neuropsychiatric diseases. Yellow colour shapes indicate different types of zinc finger domains. Note: ZNF41 contains 18 ZNF domains in cluster. 
bipolar disease, ZNF804A has also been found to associate with neurodevelopmental disorders such as dyslexia and autism $[6,23]$.

Human ZNF804A is expressed in the developing neocortex, especially during the second trimester, but not adult brain $[24,25]$ demonstrating that developmental role of ZNF804A in human brain. Knockdown of ZNF804a using shRNA in primary cultured neurons showed the disrupted neurite outgrowth, reduced dendritic spine density and activity dependent neurite outgrowth [26].

Genome-wide association study of schizophrenia patients revealed another significant association zinger finger gene: ZEB2. Different mutations in this gene cause Mowat-Wilson syndrome (MWS), an autosomal dominant disorder characterized by a number of health defects including Hirschsprung's disease, intellectual disability and epilepsy. The analysis of magnetic resonance imaging (MRI) for brains of MWS revealed the absence of corpus callosum, hippocampal abnormalities enlargement of cerebral ventricles and reduction of white matter thickness [27]. Moreover, ZEB2 is required to repress the Nkx2-1 homeobox transcription factor in the generation of cortical GABAergic interneurons. The lack of ZEB2 masks the repression of homeobox transcription factor Nkx2-1, which favors differentiation of striatal interneurons instead of cortical neurons, providing a mechanism for epilepsy in MWS [28].

ZNF41 gene has been found to be disrupted with an X-linked mental retardation (MRX). There are multiple zinc finger DNA binding motifs and KRAB-A, KRAB-B domains in ZNF41 protein (Figure 1) which act as transcriptional repressors. Expression studies in cell lines from MRX patients indicated the absence of ZNF41 transcripts, suggesting that the mental disorder in some patients may result from loss of functional ZNF41. Missense mutations as well as splice variant missing are found in affected

\section{References}

1 Laity JH, Lee BM, Wright PE (2001) Zinc finger proteins: new insights into structural and functional diversity. Curr Opin Struct Biol 11: 39-46.

2 Gamsjaeger R, Liew CK, Loughlin FE, Crossley M, Mackay JP (2007) Sticky fingers: zinc-fingers as protein-recognition motifs. Trends Biochem Sci 32: 63-70.

3 Krishna SS, Majumdar I, Grishin NV (2003) Structural classification of zinc fingers: survey and summary. Nucleic Acids Res 31: 532-550.

4 Cassandri M, Smirnov A, Novelli F, Pitolli C, Agostini M, et al. (2017) Zinc-finger proteins in health and disease. Cell Death Discov 3: 17071.

5 Takahashi H, Takahashi K, Liu F (2009) FOXP genes, neural development, speech and language disorders. Adv Exp Med Biol 665: 117-129.

6 Anitha A, Thanseem I, Nakamura K, Vasu MM, Yamada K, et al. (2014) Zinc finger protein 804A (ZNF804A) and verbal deficits in individuals with autism. J Psychiatry Neurosci 39: 294-303.

7 Sun Y, Hu D, Liang J, Bao YP, Meng SQ, et al. (2015) Association between variants of zinc finger genes and psychiatric disorders: systematic review and meta-analysis. Schizophr Res 162: 124-137. members of families with MRX [29]. The results suggested that ZNF41 is critical for cognitive development. Further functional roles of ZNF41 in corticogenesis are needed to elucidate specific mechanisms underlying MRX.

Recently, several papers reported that de novo mutation of GATAD2b gene, a subunit of the methyl-CpG-binding protein-1 complex (MECP1), causes severe intellectual disability [30-32]. GATAD2b contains only one GATA type zinc finger domain (Figure 1) is a transcription repressor through deacetylating methylated nucleosomes. It is still controversial about whether the GATA zinc finger domain mediates binding of DNA or protein interaction or both. GATAD2b is highly expressed in embryonic brain as early as E11 and adult neocortex according to mouse ENCODE transcriptome data, indicating its role in brain development as well as normal function of mature cortical neurons.

\section{Conclusion}

As genomic techniques advance recently, increasing number of zinc finger domain containing proteins have been found associated with neurodevelopmental and psychiatric diseases. Interestingly, many ZNF/zfp genes identified are transcription factors and expressed highly during embryonic brain, indicating significant roles of zinc finger domain containing proteins in brain development. As diverse as ZNF/zfp proteins, the function of $\mathrm{ZNF} / \mathrm{zfp}$ in brain development are versatile, with much remaining unknown. ZNF/zfp genes involved in cognitive development and brain development are far more than we can list here. Future studies focusing on examining spatial and temporal expression of the ZNF/zfp genes in the brain, their subcellular distribution in neurons and cross talk with other family proteins, RNA, DNA or other molecules will be needed to fully understand their roles in cognitive dysfunctions in children and adult and develop proper well-targeting therapeutic methods.

8 Ardley HC (2009) Ring finger ubiquitin protein ligases and their implication to the pathogenesis of human diseases. Curr Pharm Des 15: 3697-3715.

9 Sanchez R, Zhou MM (2011) The PHD finger: a versatile epigenome reader. Trends Biochem Sci 36: 364-372.

10 Tzchori I, Day TF, Carolan PJ, Zhao Y, Wassif CA, et al. (2009) LIM homeobox transcription factors integrate signaling events that control three-dimensional limb patterning and growth. Development 136: 1375-1385.

11 Srivastava M, Larroux C, Lu DR, Mohanty K, Chapman J, et al. (2010) Early evolution of the LIM homeobox gene family. BMC Biol 8: 4.

12 Lee SM, Li HY, Ng EK, Or SM, Chan KK, et al. (1999) Characterization of a brain-specific nuclear LIM domain protein (FHL1B) which is an alternatively spliced variant of FHL1. Gene 237: 253-263.

13 Sang M, Ma L, Sang M, Zhou X, Gao W, et al. (2014) LIM-domain-only proteins: multifunctional nuclear transcription coregulators that interacts with diverse proteins. Mol Biol Rep 41: 1067-1073.

14 Ferland RJ, Cherry TJ, Preware PO, Morrisey EE, Walsh CA (2003) Characterization of Foxp2 and Foxp1 mRNA and protein in the developing and mature brain. J Comp Neurol 460: 266-279. 
15 De Rocker N, Vergult S, Koolen D, Jacobs E, Hoischen A, et al. (2015) Refinement of the critical 2p25.3 deletion region: the role of MYT1L in intellectual disability and obesity. Genet Med 17: 460-466.

16 Mall M, Kareta MS, Chanda S, Ahlenius H, Perotti N, et al. (2017) Myt1l safeguards neuronal identity by actively repressing many nonneuronal fates. Nature 544: 245-249.

17 Cordeddu V, Redeker B, Stellacci E, Jongejan A, Fragale A, et al. (2014) Mutations in ZBTB20 cause Primrose syndrome. Nat Genet 46: 815-817.

18 Davies MN, Krause L, Bell JT, Gao F, Ward KJ, et al. (2014) Hypermethylation in the ZBTB20 gene is associated with major depressive disorder. Genome Biol 15: R56.

19 Mohnke S, Erk S, Schnell K, Schütz C, Romanczuk-Seiferth N, et al. (2014) Further evidence for the impact of a genome-wide-supported psychosis risk variant in ZNF804A on the theory of mind network. Neuropsychopharmacology 39: 1196-1205.

20 Nenadic I, Maitra R, Basmanav FB, Schultz CC, Lorenz C, et al. (2015) ZNF804A genetic variation ( $r$ 1344706) affects brain grey but not white matter in schizophrenia and healthy subjects. Psychol Med 45: 143-152.

21 Wei Q, Li M, Kang Z, Li L, Diao F, et al. (2015) ZNF804A rs1344706 is associated with cortical thickness, surface area, and cortical volume of the unmedicated first episode schizophrenia and healthy controls. Am J Med Genet B Neuropsychiatr Genet 168B: 265-273.

22 Wang J, Zhao S, Shugart YY, Zhou Z, Jin C, et al. (2016) No association between ZNF804A rs1344706 and schizophrenia in a case-control study of Han Chinese. Neurosci Lett 618: 14-18.

23 Becker J, Czamara D, Hoffmann P, Landerl K, Blomert L, et al. (2012) Evidence for the involvement of ZNF804A in cognitive processes of relevance to reading and spelling. Transl Psychiatry 2: e136.

24 Bernstein HG, Steiner J, Dobrowolny H, Bogerts B (2014) ZNF804A protein is widely expressed in human brain neurons: possible implications on normal brain structure and pathomorphologic changes in schizophrenia. Schizophr Bull 40: 499-500.

25 Chang EH, Kirtley A, Chandon TS, Borger P, Husain-Krautter S, et al. (2015) Postnatal neurodevelopmental expression and glutamatedependent regulation of the ZNF804A rodent homologue. Schizophr Res 168: 402-410.

26 Deans PJM, Raval P, Sellers KJ, Gatford NJF, Halai S, et al. (2016) Psychosis risk candidate ZNF804A localizes to synapses and regulates neurite formation and dendritic spine structure. Biol Psychiatry 82: 49-61.

27 Garavelli L, Ivanovski I, Caraffi SG, Santodirocco D, Pollazzon M, et al. (2017) Neuroimaging findings in Mowat-Wilson syndrome: a study of 54 patients. Genet Med 19: 691-700.

28 McKinsey GL, Lindtner S, Trzcinski B, Visel A, Pennacchio LA, et al. (2013) Dlx1\&amp;2-dependent expression of Zfhx1b (Sip1, Zeb2) regulates the fate switch between cortical and striatal interneurons. Neuron 77: 83-98.

29 Shoichet SA, Hoffmann K, Menzel C, Trautmann U, Moser B, et al. (2003) Mutations in the ZNF41 gene are associated with cognitive deficits: identification of a new candidate for X-linked mental retardation. Am J Hum Genet 73: 1341-1354.

30 Willemsen $\mathrm{MH}$, Nijhof B, Fenckova M, Nillesen WM, Bongers EM, et al. (2013) GATAD2B loss-of-function mutations cause a recognisable syndrome with intellectual disability and are associated with learning deficits and synaptic undergrowth in Drosophila. J Med Genet 50: 507-514.

31 Hamdan FF, Srour M, Capo-Chichi JM, Daoud H, Nassif C, et al. (2014) De Novo mutations in moderate or severe intellectual disability. PLoS Genet 10: e1004772.

32 Luo X, Zou Y, Tan B, Zhang Y, Guo J, et al. (2017) Novel GATAD2B lossof-function mutations cause intellectual disability in two unrelated cases. J Hum Genet 62: 513-516. 